\title{
Measurement the Spectroscopic Temperature of Electron in Aluminum Laser Induced Plasma
}

\author{
Mohammed R. Abdulameer \\ Department of Physics, College of Science, University of Baghdad, Baghdad-Iraq. \\ Corresponding Author: mohammed_plasma@scbaghdad.edu.iq
}

\begin{abstract}
In this research, optical emission spectroscopic studies have been carried out in Aluminum plasma generated using $1064 \mathrm{~nm}, 10 \mathrm{~ns}$ pulses from Nd:YAG laser. Temperature was estimated from the analysis of spectral data. The temperature values measurements have been performed by the comparison of two selected spectral lines of the radiation emitted by laser induced plasma of $\mathrm{Al}$ using singly ionized $\mathrm{Al}$ atom and neutral lines. An initial temperature of $2.6 \mathrm{eV}$ was measured. The relation between electron temperature and laser pulse energy in the laser-generated Al plasma have been analyzed. The electron temperatures values are found to increase from $2.6 \mathrm{eV}$ to $2.8 \mathrm{eV}$ then to $2.9 \mathrm{eV}$ as increasing laser pulse energy. [DOI: 10.22401/JNUS.20.2.10]
\end{abstract}

Keywords: Laser induced plasma, Spectrometer, electron temperature, plasma physics, plasma plume.

\section{Introduction}

The interaction of laser pulses with solids, liquids and gases had many technological applications ranging from laser processing of solids, laser-induced mass spectrometry to film deposition by pulsed lasers and has gained currency in both fundamental and applied studies.[1-4]. Laser-created plasma characteristics are strongly dependent upon several key parameters, including laser intensity،pulse duration and wavelength, target material and geometry،and the nature and pressure of any ambient gas.[5,1,3]. The laser energy transferred to target material through electrons involves different mechanisms, viz. electron-phonon coupling, multi-photon ionization and cascade ionization, etc. [3]. The laser induced evaporation and ionization is separated into three domains: (i) heat conduction inside the solid, (ii) the evaporation wave attached to the solid surface, and (iii) expansion of the high temperature vapor [6]. By increasing the fluence over the ablation threshold, the saturation of the produced plasma density allows the increase of the ions and electrons temperatures [7]. Because of the transient features of the plasma created by LIP, optical emission spectroscopy (OES) technique with time and space resolution is especially used to obtain information about the behavior of the created plasma species in space and time as well as the dynamics of the plasma evolution [8-10]. The ratio for intensity of two lines of the same species of ionization stage $\mathrm{Z}$ in the emission spectrum of the target ablated $b$ laser is expressed as:

$\frac{I_{1}}{I_{2}}=\left(\frac{\lambda_{k i, Z}}{\lambda_{n m, Z}}\right)\left(\frac{A_{k i, Z}}{A_{n m . Z}}\right)\left(\frac{g_{k, Z}}{g_{n, Z}}\right) \exp \left(-\frac{E_{k, Z}-E_{n, Z}}{k_{B} T_{e}}\right)$

where $I_{1}$ is the line intensity from the $k-i$ transition (arbitrary unit), $I_{2}$ is that from the $n-$ $\mathrm{m}$ transition (arbitrary unit), $\boldsymbol{\lambda}_{\boldsymbol{k} \boldsymbol{i}, \boldsymbol{Z}}$ is the wavelength from the $\mathrm{k}-\mathrm{i}$ transition $(\mathrm{nm})$, $\lambda_{n m, Z}$ is the wavelength from the $n-m$ transition (nm), $\boldsymbol{A}_{\boldsymbol{k} i, \boldsymbol{Z}}$ is the transition probability from the $\mathrm{k}-\mathrm{i}$ transition $\left(\mathrm{s}^{-1}\right), \boldsymbol{A}_{\boldsymbol{n} m \cdot \boldsymbol{Z}}$ is the transition probability from the $n-m$ transition $\left(\mathrm{s}^{-1}\right), \boldsymbol{g}_{\boldsymbol{k}, \boldsymbol{Z}}$ is the statistical weight of the k state, $\boldsymbol{g}_{\boldsymbol{n}, \boldsymbol{Z}}$ is the statistical weight of the n state, $\boldsymbol{E}_{\boldsymbol{k}, \boldsymbol{Z}}$ is the energy of the k state $(\mathrm{eV})$, $\boldsymbol{E}_{\boldsymbol{n}, \boldsymbol{Z}}$ is the energy of the n state $(\mathrm{eV}), \mathrm{k}_{\mathrm{B}}$ is the Boltzmann constant $1.38 \times 10^{-23} \mathrm{~J}^{-\mathrm{K}^{-1}}$, and $\mathrm{T}_{\mathrm{e}}$ is the electron temperature $(\mathrm{eV})$. From equation (1) we can find the electron temperature $T_{e}$ by comparison of two spectral lines in the emission spectrum [10]. The aim of this research is to estimate electron temperature $T_{e}$ of plasma produced by $\mathrm{Nd}$ :YAG pulsed laser of $\mathrm{Al}$ element which can be used in many application such as electroptics devices and semiconductors alloys. 


\section{Experimental Work}

The target of the laser induced plasma (LIP) process was Al with purity $99.999 \%$, and compressed under the pressure (10 tons) in order to make it shaped liked disc with about a diameter of $3 \mathrm{~cm}$ and then sintered in the oven to temperature $\mathrm{T}\left(400 \mathrm{C}^{0}\right)$ for two hours. LIP experiment was achieved under vacuum pressure 0.1mbar by using Varian DS219 Rotary pump. The beam of Nd: YAG laser with fundamental harmonic frequency ( $\lambda=1064 \mathrm{~nm}, 10 \mathrm{~ns}, 6 \mathrm{~Hz}$ ) was focused onto $\mathrm{Al}$ target with quartz lens $\mathrm{f}=10 \mathrm{~cm}$, the target was kept onto a rotating motor at a speed $4 \mathrm{rev} / \mathrm{min}$ to prevent fast drilling. The spectroscope used to collect the emission radiation from the plasma was of the type Surwit S3000-UVNIR. The LIP experiment was performed at room temperature. LIP setup scheme has been shown in Fig.(1). Electron temperature of the $\mathrm{Al}$ ablated atoms were calculated by analyzing the emission line spectra of the spectroscope data. The Al target was ablated by 1000 pulses. The vacuum pressure was varied from 0.04 to 0.2 mbar, also the laser pulse energy was selected to be 800,860 , and $940 \mathrm{~mJ}$ to study their effects on the value of electron temperature.

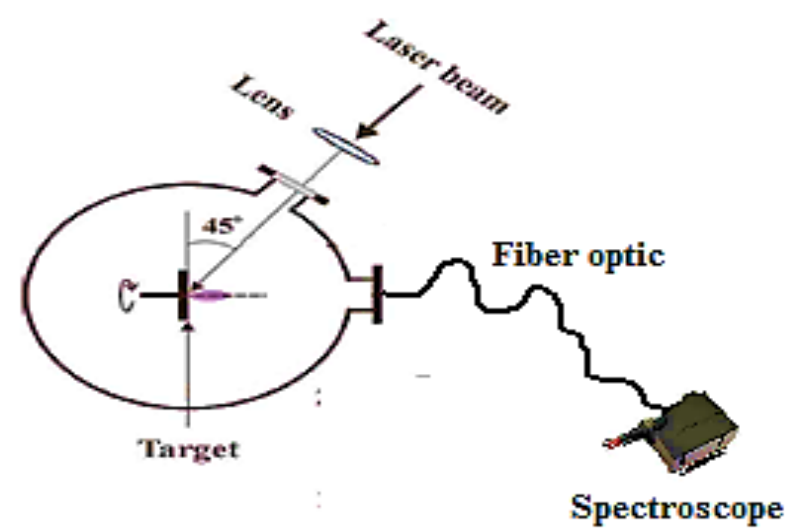

Fig.(1): Schematic diagram of the LIP experimental setup.

\section{Results and Discussion}

Different laser pulse energies 800,860, and $940 \mathrm{~mJ}$ on Al sample has been done, and by using the spectrometer and comparing with NIST database a certain peaks as shown in table 1 has been taken from the figure between the wavelength and the intensity as in figures 2,3 , and 4 .

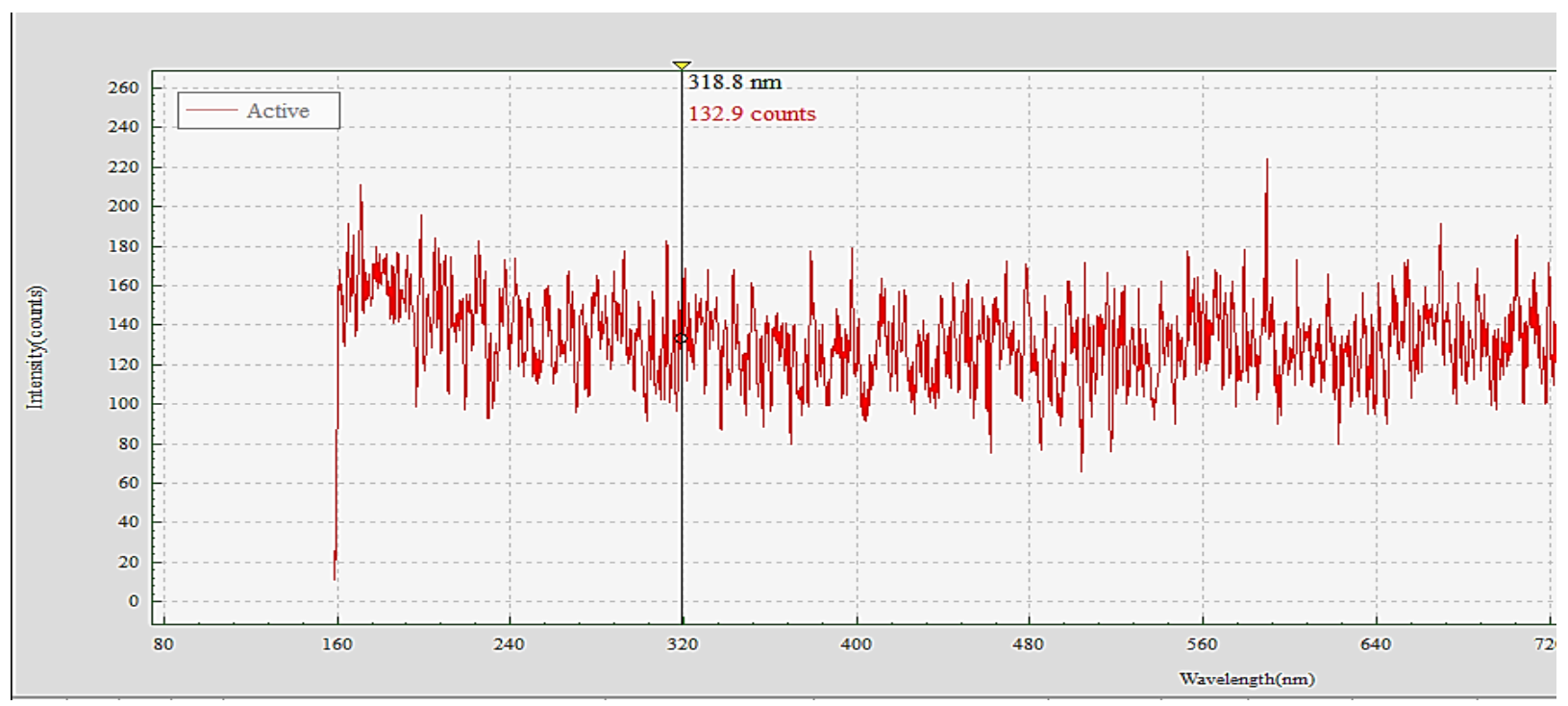

Fig.(2): Emission spectrum of Al plasma at laser pulse energy 940mJ. 


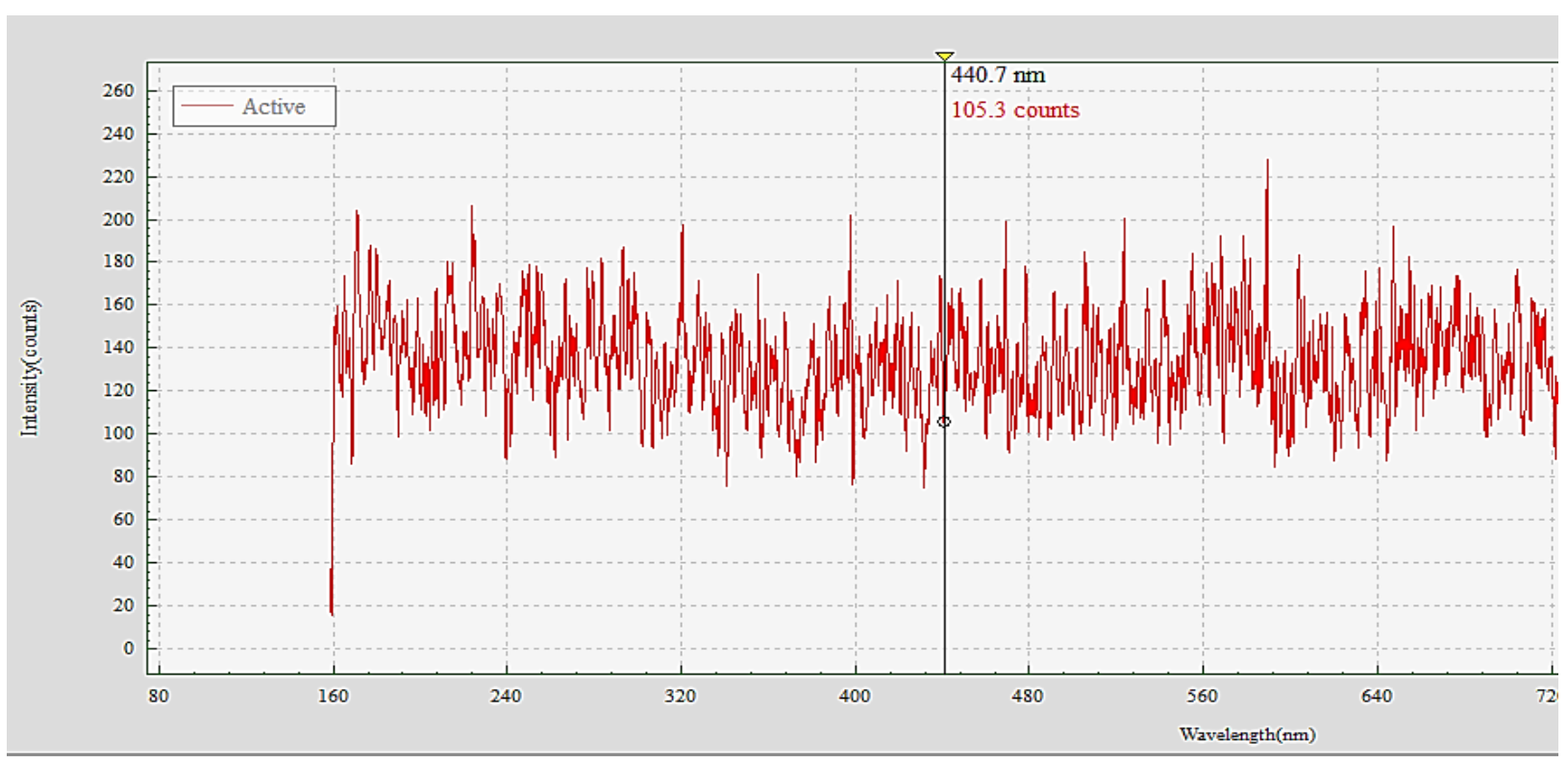

Fig.(3): Emission spectrum of Al plasma at laser pulse energy $860 \mathrm{~mJ}$.

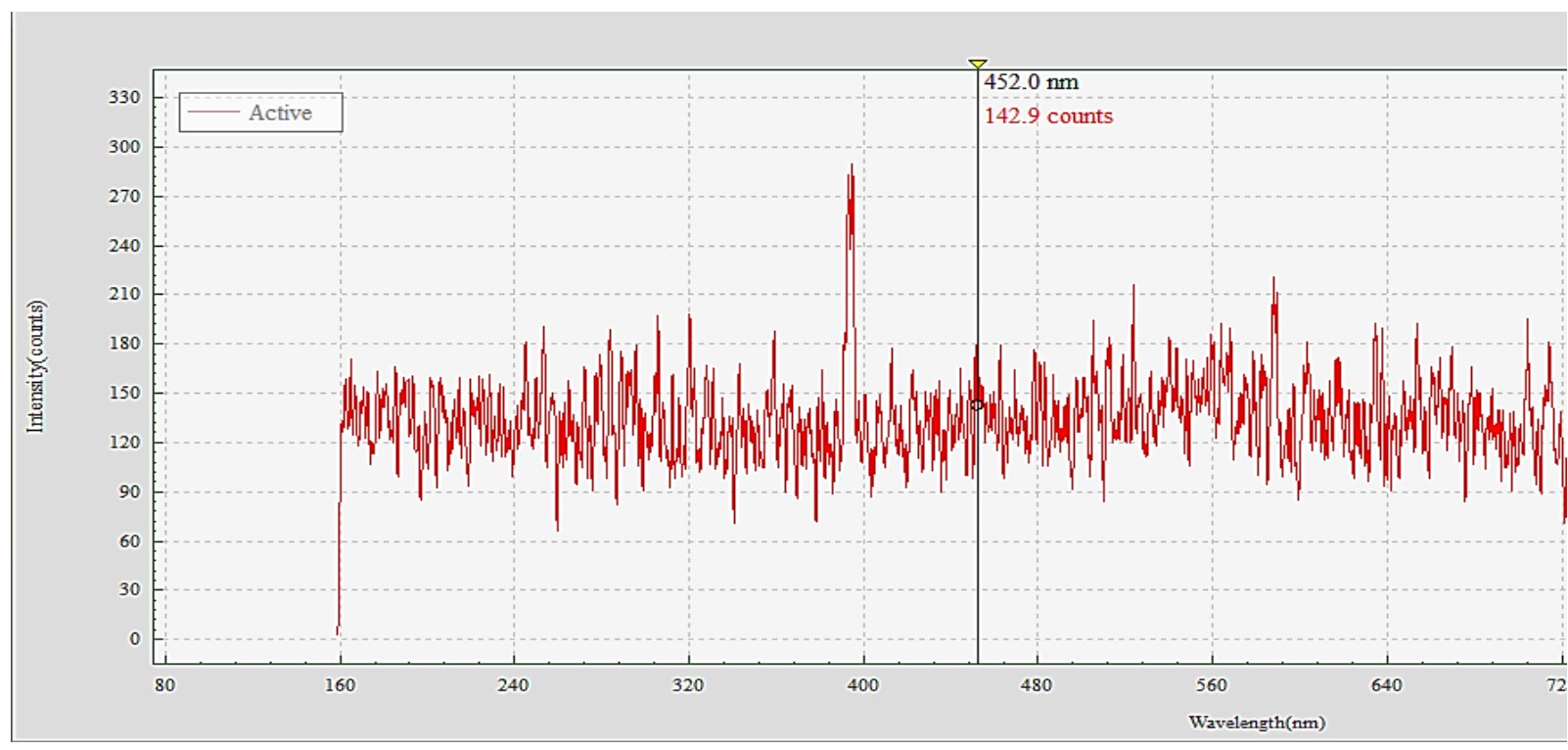

Fig.(4): Emission spectrum of Al plasma at laser pulse energy $800 \mathrm{~mJ}$.

The electron temperature can be calculated as shown in Table (1).

Table (1)

Experimental and result data.

\begin{tabular}{|c|c||c|c|c|c||c|c|c|c||}
\hline $\mathbf{E}_{\text {Laser }}$ & $\mathbf{I}_{\mathbf{1}}$ & $\mathbf{I}_{\mathbf{2}}$ & $\mathbf{g}_{\mathbf{k}} \mathbf{A}_{\mathbf{k i}}$ & $\mathbf{g}_{\mathbf{n}} \mathbf{A}_{\mathbf{n m}}$ & $\boldsymbol{\lambda}_{\mathbf{k i}}$ & $\boldsymbol{\lambda}_{\mathbf{n m}}$ & $\mathbf{E}_{\mathbf{n}}$ & $\mathbf{E}_{\mathbf{k}}$ & $\mathbf{T}_{\mathbf{e}}$ \\
\hline \hline 800 & 174.9 & 145.4 & $2.57 \times 10^{6}$ & $8.24 \times 10^{7}$ & 209.9 & 214.5 & 0 & 5.94 & 2.6 \\
\hline \hline 860 & 153.6 & 179.3 & $1.95 \times 10^{8}$ & $8.24 \times 10^{7}$ & 209.3 & 214.5 & 0.014 & 5.933 & 2.8 \\
\hline 940 & 139.9 & 159.4 & $7.75 \times 10^{8}$ & $8.24 \times 10^{7}$ & 208.5 & 214.5 & 0.014 & 5.919 & 2.9 \\
\hline
\end{tabular}

The proportional relation between the laser pulse energies and the electron temperature has been shown in Fig.(5). 


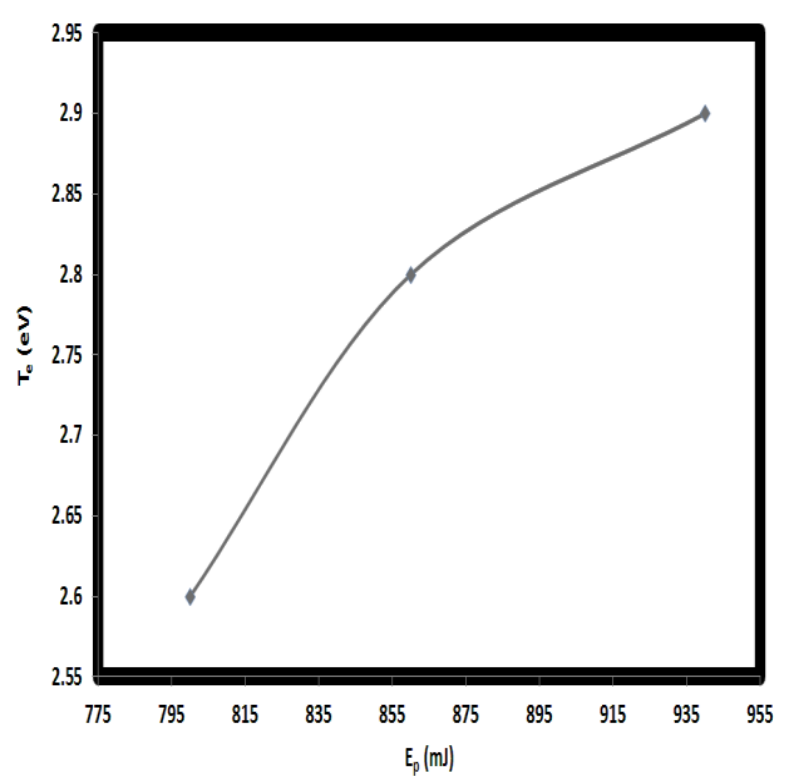

Fig.(5): Laser pulse energy as a function of the electron temperature.

Fig.(5) shows the relation between the electron temperature and laser pulse energy. It is noted from Fig.(5) that the temperature of electron increases remarkably with increasing energy because at this energy level higher energy is transferred.

These results and behavior agree with results and behavior of D. M. Surmick and C. G. Parigger as in [11]. The area nearby the surface of the target material will absorb radiation during the exposition time of laser pulse, in charge of enabling the electron to gain temperature. The high value of the electron temperature close to the surface belongs to the absorption of laser pulses by inverse bremsstrahlung absorption mechanism. Such manner of the electron temperature in Fig.(5) is due to the absorption or reflection of the laser pulse energy by plasma and relies on the frequency of plasma. It is noted also extra excited species, such as ion and free electrons because of increasing laser intensity. The laser pulse interacts with ions and free electrons and more heating, ionization and exhaustion of the arriving laser energy have been got. The electron temperature $T_{e}$ has been calculated by using eq.(1) by comparing the spectral lines $318.8 \mathrm{~nm}, 440.7 \mathrm{~nm}$ and $452 \mathrm{~nm}$ which belong to AlII , Al IV, and Al III respectively.

\section{Conclusions}

The influence of Q-switched Nd:laser on Al plasma produced by the principle harmonic wavelength on electron temperature and its manner as a function of laser pulse energy on the created plasma. The results disclose that the temperature of electron increases as laser pulse energy increases. The relation between them is directly proportional. The technique used for finding electron temperature is spectroscopic method by comparing two atomic line of $\mathrm{Al}$ target plasma induced by laser, from the comparison between these two lines electron temperature $\mathrm{T}_{\mathrm{e}}$ has been found.

\section{Acknowledgments}

Thanks to the Physics department in University of Baghdad/ Plasma laboratory for providing the apparatus and tools for this experiment.

\section{References}

[1] Bhatti K. A., Khaleeq-ur-Rahman M., Rafique M. S., Latif A., and Parveen N., "Electrical diagnostics of laser ablated platinum plasma", Vacuum, 82, 1157-1161, 2008.

[2] Hussein A. E., Diwakar P. K., Harilal S. S., and Hassanein A., "The role of laser wavelength on plasma generation and expansion of ablation plumes in air", journal of applied physics, 113, 1-10, 2013.

[3] Bhatti K A., Khaleeq-ur-Rahman M., Rafique M. S., Chaudhary K. T., and Latif A., "Electrons emission from laser induced metallic plasmas", Vacuum, 84, 980-985, 2010.

[4] Mohamed A. Khater, "Influence of laser Pulse energy on VUV emission from laser plasmas under various ambient conditions", Rom. Journ. Phys., 58, 181-192, 2013.

[5] Harilal S. S., Beau O'Shay, and Mark. S. Tillack, "Spectroscopic characterization of laser-induced tin plasma", Journal of applied physics, 98, 1-7, 2005.

[6] Zhaoyan Zhang, Zhen-Xue Han, and George S. Dulikravich, "Numerical simulation of laser induced plasma during pulsed laser deposition", Journal of applied physics, 90, 5889-5897, 2001.

[7] Tudisco S., Mascali D., Gambino N., Anzalone A., Gammino S., Musumeci F., Scordino A., and Spitaleri A., "Investigation of laser-produced aluminum plasma", Nuclear Instruments and Methods in Physics Research A, 653, 47-51, 2011. 
[8] Camacho J. J., Díaz L., Santos M., Juan L. J., and Poyato J. M. L., "Time-resolved optical emission spectroscopy of laserproduced air plasma", Journal of applied physics, 107, 1-9, 2010.

[9] Camacho J. J., Diaz L., and Poyato J. M. L., "Time-resolved spectroscopic diagnostic of laser-induced plasma on germanium targets", Journal of applied physics, 109, 15, 2011.

[10] Thiyagarajan M., and Scharer J., "Experimental investigation of ultraviolet laser induced plasma density and temperature evolution in air", Journal of applied physics, 104, 1-12, 2008.

[11] Surmick D. T., and Parigger C. G., "Time-resolved aluminum laser-induced plasma temperature measurements", Journal of physics, 548(01), 1-4, 2014. 